\title{
Confusions regarding stochastic fluctuations and accumulators in spontaneous movements
}

\author{
Carsten Bogler ${ }^{1}$, Bojana Grujičić ${ }^{2,3}$, John-Dylan Haynes ${ }^{1,2,3,4,5,6,7,{ }^{*}}$
}

1. Bernstein Center for Computational Neuroscience, Charité-Universitätsmedizin Berlin, Charitéplatz 1, 10117 Berlin, Germany

2. Max Planck School of Cognition, Stephanstr. 1A, 04103 Leipzig, Germany

3. Berlin School of Mind and Brain, Humboldt-Universität zu Berlin, Unter den Linden 6, 10099 Berlin, Germany

4. Berlin Center for Advanced Neuroimaging, Charité-Universitätsmedizin Berlin, Charitéplatz 1, 10117 Berlin, Germany

5. Clinic of Neurology, Charité-Universitätsmedizin Berlin, Charitéplatz 1, 10117 Berlin, Germany

6. Institute of Psychology, Humboldt-Universität zu Berlin, Unter den Linden 6, 10099 Berlin, Germany

7. Cluster of Excellence "Science of Intelligence", Berlin Institute of Technology, Marchstr. 23, 10587 Berlin, Germany

\footnotetext{
* Please address correspondence to: John-Dylan Haynes, Bernstein Center for Computational Neuroscience, Charité-Universitätsmedizin Berlin, Charitéplatz 1, 10117 Berlin, Germany. Email: haynes@bccn-berlin.de.
} 


\section{ABSTRACT}

Experiments on choice-predictive brain signals have played an important role in the debate on free will. In a seminal study, Benjamin Libet and colleagues found that a negative-going EEG signal, the readiness potential (RP), can be observed over motor-related brain regions already a few hundred ms before a participant makes a conscious decision to move. If the onset of the readiness potential is taken as an indicator of the "brain's decision to move" this could mean that this decision to move is made early, by unconscious brain activity, rather than later, at the time when the subject believes to be deciding. However, an alternative interpretation has recently been discussed, the stochastic decision model (SDM), that takes its inspiration from models of perceptual decision making. It suggests that the RP originates from an accumulation of stochastic internal fluctuations. In this view the decision happens only at a much later stage when an accumulated noise signal reaches a threshold. Here we address a number of confusions regarding both the evidence for the stochastic decision model as well as its interpretation. We will show: (a) that the evidence for the role of stochastic fluctuations is highly indirect; (b) that there is little direct support for the SDM from animal studies; (c) that deterministic (non-random) processes can explain the data in a similar way; (d) that the relative components of the model have been mischaracterized leading to an over-emphasis on the role of random fluctuations and an under-emphasis of deterministic aspects of the model; (e) that there is confusion regarding the role of "urgency" and "evidence" in the SDM and its link to perceptual decision making; (f) that the question whether the decision happens early or late depends on the nature of the noise fluctuations, specifically, whether they reflect "absolute" or "epistemic" randomness; (g) finally, that the model does not explain the temporal relationship between conscious decision and neural decision. Our aim is not to rehabilitate the role of RPs in the free will debate. Rather we aim to address some confusions and premature conclusions regarding the evidence for accumulators playing a role in these preparatory brain processes. 


\section{Introduction}

Throughout the day we have to make a multitude of decisions about external stimuli. For example, when we see a car crossing our lane on the highway we step on the break to avoid a collision. An important factor is the level or quality of sensory information. For example, when driving in broad daylight we instantly see the dangerous car. But when it is foggy we might be uncertain about whether it is a car or just a random pattern in the mist. In that case we might need to look at the pattern for a bit longer and gather evidence across time. A popular approach for explaining perceptual decision making (PDM) under such varying levels of sensory evidence is the accumulator model (Smith \& Ratcliff, 2004). It formulates a mechanism that accumulates sensory evidence across time and thus gradually improves the accuracy of a sensory decision. When the buildup of evidence crosses a set threshold the decision is reached and a reaction can be triggered. Most accumulator models involve two key variables that are combined in an additive fashion: the first term is the sensory evidence in each time step that reflects a single sample of information about the external stimulus; the second term is internal noise that accounts for variability in responses. The accumulator adds both terms, the evidence and the noise, as inputs to its ongoing total evidence tally. So both the external evidence and the internal noise contribute to the decision. When the external information is high (as in broad daylight) the process is dominated by the evidence. When the external information is low (as in fog) the process is dominated by the internal noise. Sometimes, there is also a leak term so that the total evidence slowly decays if it is not refreshed (Usher \& McClelland, 2001).

In recent years, this approach has also been used to explain the neural mechanisms underlying simple, self-paced motor decisions (Schurger, 2018; Schurger et al., 2012). These movement decisions have been met with considerable interest in debates of free-will and volition. This is because such spontaneous decisions are preceded by a slow negative-going EEG signal, the so-called readiness potential (RP)(Kornhuber \& Deecke, 1965) that appears to occur even before a person makes a conscious decision to move (Libet et al., 1983). To give a very rough summary, a debate has centered on the following notion: if the brain "knows" that a decision will occur before a participant has consciously made up their mind, then this might mean that the decision has happened before the conscious mind kicked in, which some would consider to undermine that humans have free will. Here, we will not be interested in the free will debate, but in the mechanisms that occur before a self-paced movement occurs. Conventionally, the onset of the RP is interpreted as "postdecisional", meaning that it is triggered after the decision to move has been made by the brain. In that view the early onset of the RP reflects an early decision of the brain that happens before consciousness kicks in. 
Recently, an alternative explanation has been proposed that is based on an accumulator model and that places the time of the decision at a much later time. As we will see, a key difference here is that the RP - rather than being postdecisional - reflects a predecisional stage where the decision has not yet been made and during which the brain activity is governed by random fluctuations. More specifically, this stochastic decision model (SDM) proposed by Schurger et al. (2012) takes its inspiration from the leaky accumulator model that is well-established in perceptual decision making (Usher \& McClelland, 2001). However, now the noise term from the accumulator takes center stage. The idea is that the decision is determined largely by the accumulation of the random internal fluctuations. Fluctuation-based accounts have long been used to explain Libet's findings (Eccles, 1985; Libet, 1985; Ringo, 1985; Stamm, 1985). These older accounts do not explicitly employ accumulators, but slowly fluctuating signals (note: a leaky accumulator is very similar to a low-pass filter and generates similar slow fluctuations when provided with noisy input).

In the SDM, the accumulated fluctuations alone would not suffice to drive the signal over the decision bound within reasonable time, so an additional process is required that brings the process into the operating range close to the decision bound so that the accumulated internal fluctuations can spuriously drive the signal across the boundary:

"In our model this solution amounts to simply shifting premotor activation up closer to the threshold for initiation of the instructed movement and waiting for a random threshold-crossing event." (Schurger et al., 2012, p. E2905).

In the SDM, this process that drives the signal closer to the threshold is called an "urgency" signal. It is necessary to prevent having to wait for a very long time for the decision (see below). Interestingly, this urgency signal is mathematically equivalent to the "evidence" signal in perceptual decision making, but it has a very different interpretation.

In the following our primary aim is to clarify several points regarding the SDM that have led to confusions in the literature. While many authors correctly cite and discuss the architecture and the implications of the model, there still seem to be a lot of misunderstandings regarding several aspects. Our interest here is not to fully review the literature on the readiness potential, to re-introduce the readiness potential into the debate on free will, or to finally rule out the accumulator model as a potential mechanism for spontaneous actions. We rather aim to delineate more clearly what the SDM-related findings mean and what they don't mean. We will provide some equations, but this paper should be approachable even without an in-depth understanding of the mathematical foundations. Readers who do not want to dive into the mathematical basics can jump over the next section. 


\section{How does the accumulator model work?}

Now is the time to go into more detail how the model works and which evidence is provided in support of it. We will focus on two papers, Schurger et al. (2012) and Schurger (2018), because these contain explicit mathematical formulations. They are both similar variants from the larger family of accumulator models (e.g. Smith \& Ratcliff, 2004). We will reorder some aspects for easier readability and we will use difference equations rather than their differential variants because all their modelling is done in discrete time steps $i$.

The key variable is the accumulated signal $x_{i}$ (Fig. 1, left). At the beginning of the trial this variable starts at $\mathrm{x}_{0}=0$ (a starting bias is not used). On every time step an increment or decrement $\Delta x_{i}$ is added to $x_{i}$ and when $x_{i}$ reaches a threshold $\beta$ a movement is triggered at time point $T$ also referred to as waiting time. (Please note: In a realistic brain there will still be a delay between the time T when the movement command is sent into the motor system, say down the spinal cord, and the time when the movement begins in the muscles; the original paper, Schurger et al., 2012, acknowledges this, but then their model implementation ignores this latency, presumably for simplicity; we will thus also ignore it here).

In each time step $\mathrm{x}_{\mathrm{i}}$ is updated by $\Delta x_{i}$ based on the following equation (rewritten from the original in a slightly more explicit form):

$$
\Delta x_{i}=I \Delta t+c \xi_{i} \sqrt{\Delta t}-k x_{i} \Delta t
$$

This means that the increment/decrement that is added to $x_{i}$ on each trial depends on three additive components:

1. The first term, $I \Delta t$, is a constant that is referred to as "urgency" in the SDM. This is mathematically equivalent to the (mean) evidence in accumulator models of perceptual decision making, but it has a fundamentally different interpretation.

2. The second term, $c \xi_{i} \sqrt{\Delta t}$, reflects internal Gaussian noise $\xi_{i}$ that is scaled by $c$ and $\sqrt{\Delta t}$ (in the SDM both $\Delta t$ and $\mathrm{c}$ are used as fixed scaling constants).

3. The third term, $k x_{i} \Delta t$, reflects the leak term with the leak constant $k$ scaled with another constant $\Delta t$. Thus, the accumulated signal is reduced by a constant proportion of $x_{i}$ on each time step.

When the accumulated signal $\mathrm{x}_{\mathrm{i}}$ crosses the threshold $\beta$, a motor command is triggered. Figure 1 (left) shows the operation of the model expressed as a more conventional box and arrows model. There, the three inputs from above are shown as arrows feeding into the accumulator. Let's look at the behavior of a single trial (Fig. 1, top right; Fig. 1, bottom right, shows this process for a large number 
of trials). In every trial the accumulation starts at the first step at $x_{0}=0$ (other accumulator models sometimes introduce a starting bias here). In every time step the increment (or decrement) $\Delta x_{i}$ is added to $x_{i}$, resulting in a noisy drift towards the decision boundary. At some point the accumulated signal crosses the decision boundary (Fig. 1, top right, dashed line) and triggers a response at latency T.

The following two rows of the figure also show the consequences of removing the urgency (i.e. the constant) term versus the noise term. If the noise term is removed the signal $x_{i}$ rises and depending on the parameters of the model asymptotes below the threshold (as shown here, with the parameters in Schurger et al., 2012) or it crosses the threshold (Schurger, 2018). If the urgency term is removed the signal meanders around for a long time and at some point crosses the threshold, but with an implausibly long latency (for details see Fig. 2).
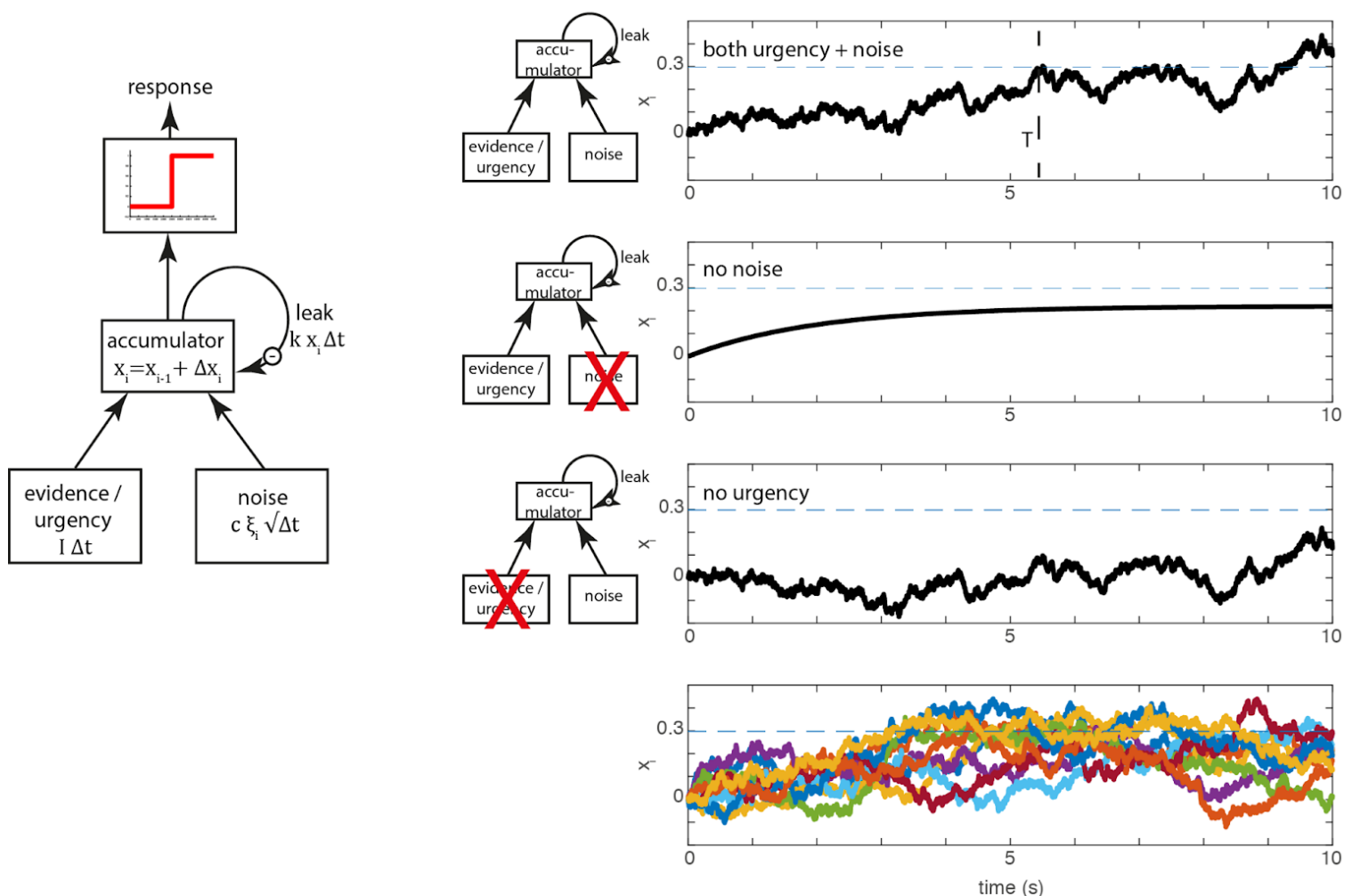

Fig. 1: Basic accumulator model. Left: In each time step in perceptual decision making, two variables, evidence and noise are added to a leaky accumulator. When the output of the accumulator reaches a certain threshold (red) a report is triggered. In spontaneous movement, the evidence is replaced by an urgency term that is added to bring the signal into a range near the threshold. Right: Examples of the stochastic decision model (SDM) (Schurger et al., 2012). The first three rows on the right show the behavior of the model in a single trial, separately for the full model (top), only the evidence/urgency with noise removed (second row) and only the noise (third row). The dashed 
horizontal line is the threshold $\beta$. The bottom row shows 10 trials, which clearly highlights the variability in individual trial accumulator trajectories. Note that in this original model, the urgency alone will not drive the accumulator beyond the threshold, and the noise alone will take an implausibly long time to drive the accumulator over the bound (see Fig. 2). Both terms together bring the signal across the threshold (at $\mathrm{T}$, top row), which then triggers a movement with a distribution of reaction times that matches the waiting times of the participants until they press the button. Please note, the compressive nature of the accumulation signal that is caused by the leak term leads to progressively shallower slopes the larger $\mathrm{x}_{\mathrm{i}}$ is.

\section{Differences between the spontaneous motor decision model and perceptual decision making}

The SDM for endogenous decisions is mathematically equivalent to a variant of the perceptual accumulator model (Usher \& McClelland, 2001). The two scenarios differ only regarding the interpretation of the parameter $I$. In perceptual decisions, the drift term $I$ refers to the mean sensory evidence. Normally, in perceptual decision making, the constant term, the sensory evidence, is the main driving factor towards the decision boundary. The noise component is sometimes referred to as reflecting moment-to-moment changes in evidence, but by itself the noise component does not contribute any evidence at all because it is mean-centered. In perceptual decision making, when the evidence is 0 , the behavior of the accumulator is governed by the noise term (see Fig. 1, right).

We would like to highlight two points of the accumulator model in perceptual decision making. First, in the model as formulated here, for a given evidence level the drift $I$ is a constant (see e.g. Ratcliff 1978 for variations on this assumption). It reflects the mean amount of sensory evidence that some neurons are encoding about an external stimulus property. For example, a motion stimulus of a specific coherency level on an external monitor will lead to a constant representation of momentary evidence about this motion direction in motion area MT in the brain, and this evidence is summed up by the accumulator. Second, the term "evidence" here means that the signal in MT has information about another property, the external motion stimulus. This evidence can also be very small or even 0 in case of very weak or no sensory evidence.

So how does this perceptual decision making model transfer to spontaneous movements? In a review paper Schurger and colleagues state:

"A strength of SDMs [stochastic decision models] is that they provide a unifying story that seamlessly allows agents to move between reason-driven and random decisions, as the spontaneous action case is just an SDM driven by noise in the absence of evidence/reasons." (Schurger et al., 2021, p. 10, underline added).

Based on this statement one might think that the spontaneous movement model (SDM) is based on the perceptual decision making model, but with zero evidence, with only the noise active, thus 
making it similar to perceptual guessing. This is indeed a reasonable assumption and it has been tested before. For example, we have shown previously that perceptual guesses (perceptual decisions with no sensory evidence) and spontaneous decisions elicit similar activation patterns in posterior parietal cortex (Bode et al., 2013).

However, the SDM does not do what is implied in that brief summary. The random decisions are not modelled in the same way as perceptual decisions because the drift/evidence term $I$ is not set to zero, but is given a new role as an "urgency" parameter. This is motivated by the fact that the accumulation of the fluctuating noise alone would take a very long time to cross the threshold (on average 161s, see Fig. 2). This would not match up with observed response latencies (waiting times) in these tasks. So, far from providing a unifying story, the SDM postulates an additional process that is needed that first brings $\mathrm{x}_{\mathrm{i}}$ closer to the threshold so that in a second stage random noise fluctuations have a chance of pushing the signal over the threshold in reasonable time (see below). This is where the urgency comes in. The urgency is a constant input to the accumulator that serves to bring the total signal closer to the threshold. Schurger et al. (2012) say that urgency is "inherent in the demand characteristics of the task" (p. E2911). They also say that urgency is relatively weak (what weak means here is never defined). The roles of urgency and noise in the model are frequently characterized as reflecting a two-stage sequential process:

"In our model this solution amounts to simply shifting premotor activation up closer to the threshold for initiation of the instructed movement and waiting for a random threshold-crossing event." (Schurger et al., 2012, p. E2905).

We will see more examples of this notion below and demonstrate that both claims are not quite correct: the contribution of urgency is far from weak, and the model does not reflect two sequential stages. In fact, half of the decisions happen during the early stage where the urgency effect is moving the signal closer to the threshold (see below and Fig. 6). Please note that an urgency signal is only one way to bring the accumulated signal and the threshold closer together (alternatives could potentially be changing the starting point of the accumulation process or the decision boundary itself, but see Guevara Erra et al., 2019, or progressively "collapsing" the decision boundary across time, Hawkins et al., 2015).

As we will see, there is yet another confusion that will become apparent below: it is not correct to speak about "evidence" in the spontaneous movement model, as is sometimes done (see below). The signals do not reflect evidence in any meaningful sense (other than potentially that it is informative about something further downstream, such as the reaction time). They might be biases, but they do not constitute evidence about something else. 

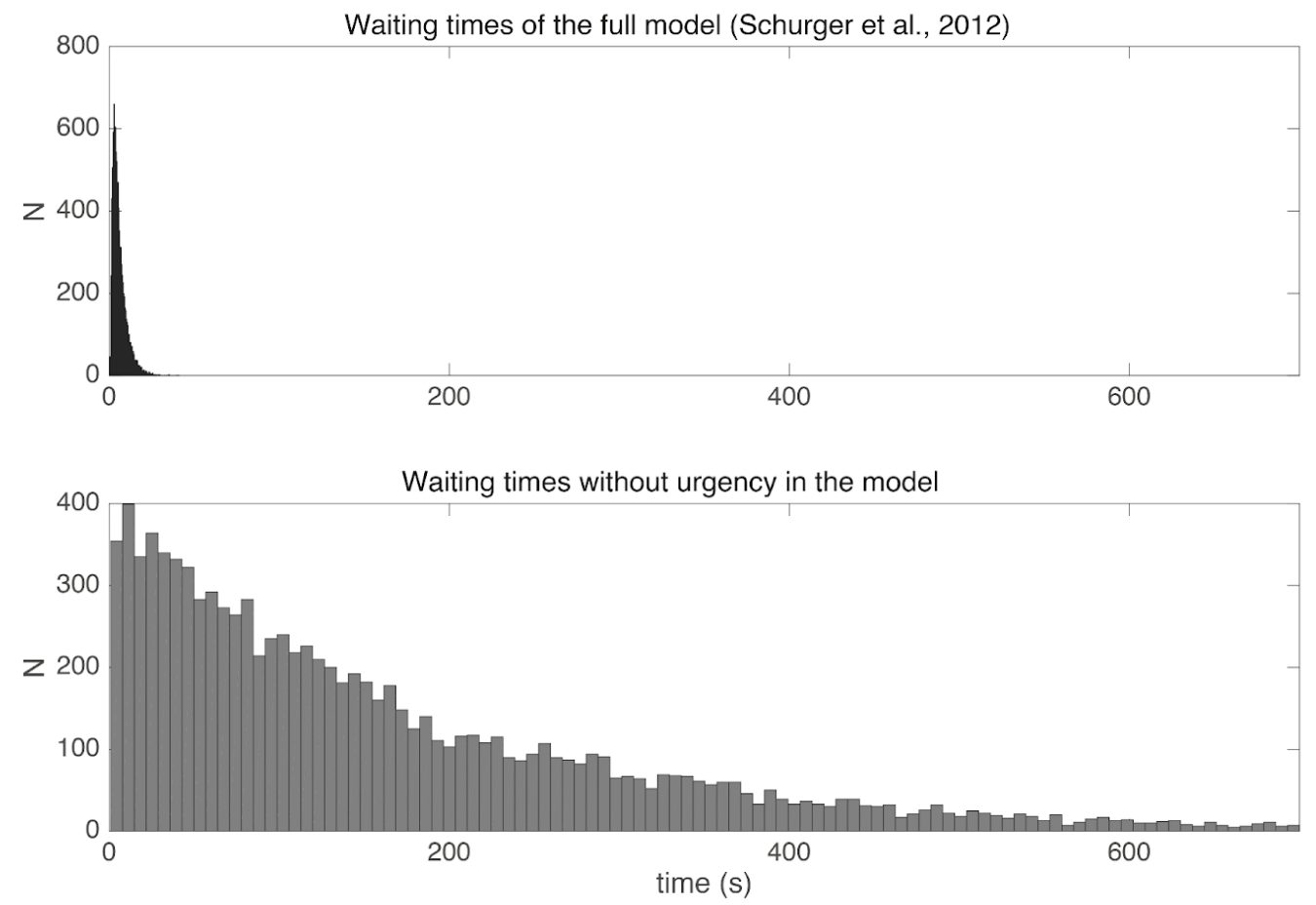

Fig. 2: The distribution of waiting times with (top) and without (bottom) the urgency parameter (same $x$-scaling to show difference). Here, the top row is generated by the top right model in Fig. 1 (from Schurger et al., 2012) and the bottom row is generated by the same model, except that the constant "urgency" factor is left out (3rd model from the top on the right of Fig. 1). Note that without the urgency parameter the waiting times are implausibly long (mean: 161s, median: 118s (bottom) vs. mean: 6s, median: $4.8 \mathrm{~s}$ (top)).

\section{How is the accumulator linked to the readiness potential?}

In order to provide support for the model, Schurger et al. (2012) show that it provides a potential explanation of the readiness potential. The idea is that the RP emerges from averaging the trajectory of the accumulated signal $x_{i}$ backwards from when it reaches the threshold (Fig. 3). Importantly, all the fitting here is done based on the average RP, i.e. by averaging across many trials (for single-trial extraction of RPs see e.g. Schultze-Kraft et al., 2016). Fig. 3 shows this principle and plots some sample trajectories using the best fitting parameters from Schurger et al. (2012). These are obtained by fitting the waiting time distribution predicted by the model to the empirical waiting time distribution observed in the behavioral data. Please note that the waiting time distributions are subject to additional transformations before being compared (Schurger et al., 2012, p. E2906). Given those specific parameters the model also predicts the readiness potential. Please note that the 
readiness potential only reflects the final stage of the modelled decision-making process. Thus, a distinction has to be made about the claims made by the entire decision making model and the claims made relating to predicting the shape of the readiness potential.
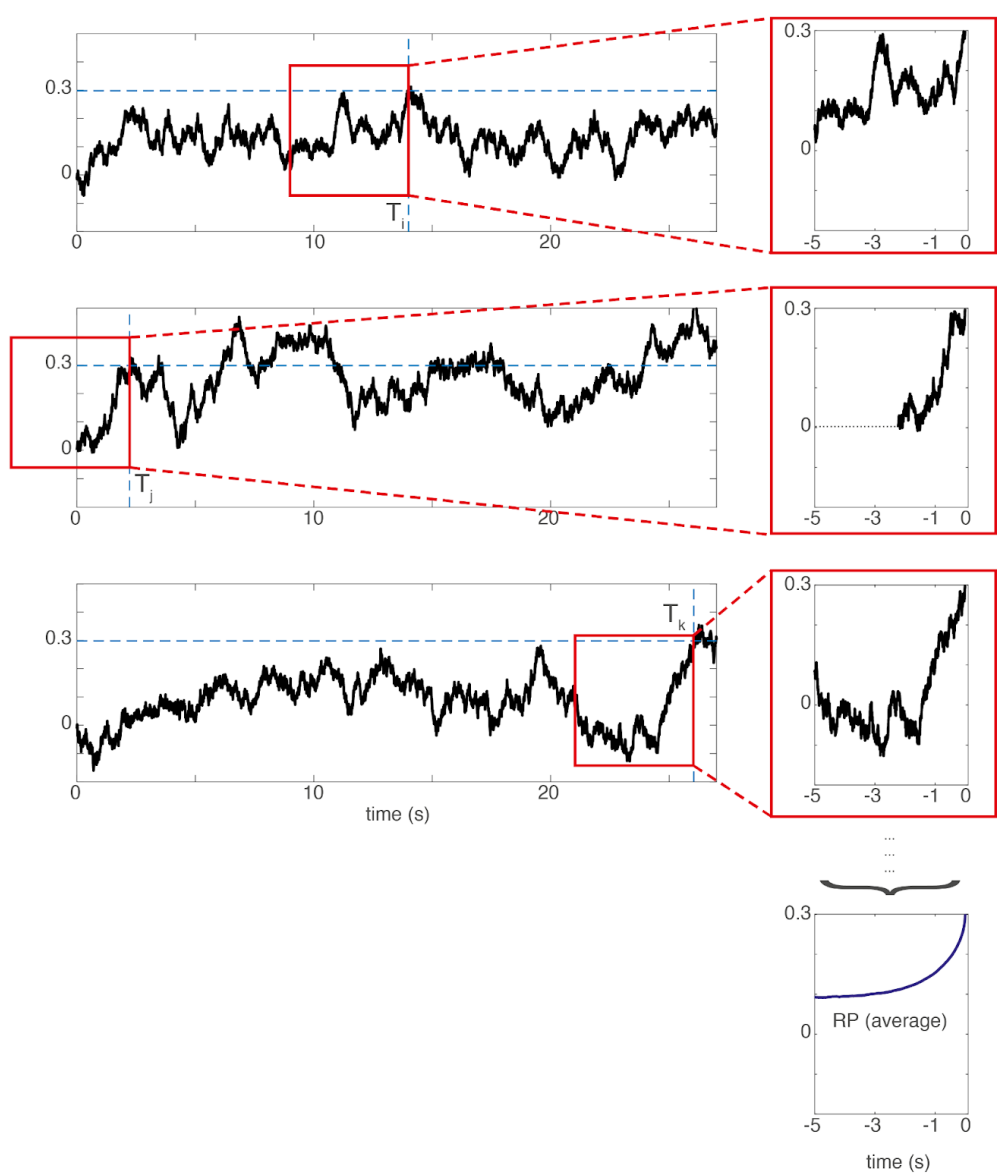

Fig. 3: The SDM assumes that the readiness potential reflects individual trajectories of the accumulated signal backwards-averaged from the time of threshold crossing $(T)$. The left shows artificially generated trajectories of a hypothetical accumulator signal $x_{i}$ in three different trials. The red box shows the 5 second averaging time window averaged backwards from the threshold crossing that can be seen on the rightmost border. The top three panels on the right show the signal in the red window enlarged and temporally aligned. The bottom right panel shows the average of these threshold-crossing-aligned trajectories across 1000 trials. This curve somewhat resembles a readiness potential. When the threshold crossing happens early in the trial (2nd row), the missing values are left out in the average (i.e. they are coded in Matlab as NaN, "not a number"). Please note that the signal trajectories are latent variables of the model, but they are not measured directly. The model fit is conducted at the level of the RP averaged across 1000 trials. Also, see (Schurger, 2018) for different assumptions underlying the spectral nature of these noise fluctuations and for different architectures of the model. Please note that the RP directly derived from the model is positive-going because the threshold is positive as in the original paper. There the time course is additionally transformed to match the empirical RP, which is negative-going. 


\section{Confusion 1: Is there direct empirical evidence for noise fluctuations?}

Next we would like to address a number of confusions that seem to have originated in the literature on the SDM. For example, summaries provided in various papers give the impression that the empirical analysis of the EEG data and of behavioral waiting times provides direct evidence for the involvement of fluctuations in the decision process, that is that they "capture these ongoing fluctuations" (Schurger et al., 2012, p. E2905). This is not the case. Instead, the fluctuations reflect a latent variable of the model that is not directly measured. The fluctuation time courses are not even inferred at a single-trial level. The time-courses are purely hypothetical and are used as part of a model to predict average properties of the recorded data. As shown above, the model fit is based solely on average behavior data and average EEG / RP signals. The gap in resolution between model variables (e.g. noise time courses of hypothetical units) and measured variables (EEG signals) is not unusual in modelling of neuroimaging data. For example dynamic causal modelling (DCM) (Friston et al., 2003) also models measured data using a number of latent variables at finer levels of temporal resolution, however this is always done at the level of raw time series data, not on averages.

To be clear: To date there is no directly measured evidence for a role of fluctuations in readiness potentials in the human brain. Thus, free choices might originate from fluctuation signals, or not, we will never know until we have invasive recordings in humans. Furthermore, there have been substantial challenges to the ubiquity and nature of another part of the SDM, that is the accumulation process, in recent years. Especially in situations where sensory evidence is brief rather than distributed across time accumulation might not always take place (Thorpe et al., 1996; Uchida \& Mainen, 2003). Furthermore, the true dynamics of information processing during decision-making might be difficult to infer from average data (e.g. Latimer et al., 2015).

\section{Confusion 2: Is the model supported by invasive recordings in animals?}

In order to provide more direct evidence the authors point to converging studies on animals. Potential evidence for the neural implementation of a SDM for endogenous tasks was reported by Murakami et al. (2014). That study investigates spontaneous movements in an intertemporal choice task in rats. After a go-signal, rats are given a choice between an immediate water reward or if they wait longer for a second signal they can obtain a higher reward. Sometimes rats wait a bit, but then spontaneously abort and go for the smaller sooner reward. These choices in "impatient trials" are somewhat endogenous because there is no immediate trigger to move. For these trials they make two observations: (a) The activity in one selectively chosen population of neurons (P1) in rat motor area $\mathrm{M} 2$ rises sharply in the last few hundred ms before the movement. They interpret this as an 


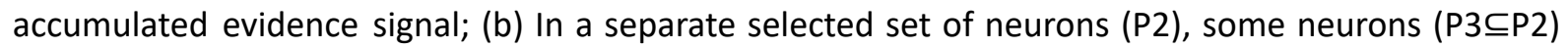
show activity that is predictive of the waiting time. At first sight the similarities could be seen as striking: the animals behave spontaneously, without any concrete immediate triggering stimulus; there is a signal $S_{\text {in }}$ that could be taken to represent the input to the accumulator and a signal $S_{\text {acc }}$ that could reflect the accumulated evidence.

A careful look, however, shows that the superficial appearance of similarity is misleading (even leaving aside the problem that it is tricky to know when exactly (or if at all) the rats make a conscious decision). There is nothing in the data to suggest that the signal $\mathrm{S}_{\text {in }}$ reflects intrinsic noise fluctuations. Instead it could simply reflect a cognitively interpretable bias signal (such as an expectation on that trial when the delayed reward will occur). Even more, however, the information in signal $\mathrm{S}_{\text {in }}$ in each neuron is transient. Thus, every neuron on its own is only informative of the waiting time in a specific brief time window, but the time windows vary across neurons (see Figs. $4 \& 5$ of Murakami et al., 2014). Even more, by inspection of Fig. 5c,d (Murakami et al., 2014) one can see that a majority of predictive time periods are around the start of the trial or even before the onset of the trial, which is the opposite of what would be expected in the case of an accumulator model. There is a considerable temporal dissociation between the time where most of the time windows are informative, and the time when the putative accumulator in their data ramps towards threshold (compare Murakami et al. 2014, Figs. 4 \& 5). If the predictive signal feeds into the accumulation one would expect it to coincide in time with the steepest increase in the accumulated signal. In their decision model, the signal from each contributing neuron is only collected in a single brief time window, and otherwise ignored (see their p. 1584). Thus, the accumulator model proposed by Murakami is dramatically different from previous models, including that by Schurger et al. (2012). One could ask whether the paper by Murakami et al. (2014) shows direct evidence of accumulation in the traditional sense at all. These findings are somewhat in contradiction with the SDM. Interestingly, the findings to some degree resemble more recent population-based models of decision making (Latimer et al., 2015). Also, finally, the Murakami task is quite different than that employed by Schurger et al. (2012), even beyond the fact that there is considerable choice-predictive information already very early in the trials. Murakami et al. use an intertemporal choice task that has a clear payoff structure, so that after some experience with the task there will be an optimal time point for switching from waiting to taking the smaller sooner reward. Thus, any signals prior to the decision could simply reflect the animal adapting to such a choice mechanism that involves e.g. keeping track of the elapsed time. 


\section{Confusion 3: Are random fluctuations an essential part of the model?}

Given that the fluctuations have not been directly measured, but only indirectly inferred, it would be interesting to know whether a simpler model, potentially without fluctuations, could in principle also generate the predictions for the RP. One such example is the linear ballistic accumulator (LBA) from perceptual decision making which is a similar accumulator model, but it works without fluctuations (Brown \& Heathcote, 2008). The LBA (Fig. 4, middle) is a simplified version of the standard accumulator model. The difference is that the drift rate $I$ (as well as a starting bias) is drawn from a random distribution only once at the beginning of each trial. Thus, it is not subject to noise fluctuations within a trial at all. The drift rate varies across trials, which could reflect e.g. differences in attention (in the case of perceptual decision making) or differences in motivation or impulsivity (in the case of spontaneous movements). In the LBA there is no within-trial noise component. Interestingly, this fluctuation-free model makes very similar predictions for features of perceptual decisions to the accumulator. Importantly, it predicts the typical heavy-tailed reaction time / waiting time distributions. When used in a similar way to predict readiness potentials, the ballistic accumulator model also provides a good fit to the RP, despite its simplicity and the absence of random fluctuations (Fig. 4). Please note that for this simple LBA model the whole process is pre-determined once the trial is started. Previously there has been the idea that at the beginning of the trial there is a general decision to move but the exact time is left open, and then subsequently during the trial there is a "decision to move now" (Schurger et al., 2012) that is the final commitment to immediately move. The LBA would constitute a different view where a decision is made at the beginning of the trial to move at time $T$ in the future.

In fact one could question whether the LBA really is an accumulator at all, despite providing a good fit of the data and carrying the label "accumulator" in its name. The answer to "when the decision is actually made" is very different for the LBA than for the SDM, because the key factor that determines the outcome is present already at the beginning of the trial. Please further note that this model is somewhat more compatible with the data by Murakami et al. (2014) because it predicts that the response time is encoded in brain signals already at the beginning of the trial. Thus, in the absence of direct tests of the link between fluctuations and RPs assuming that the decision "is made" early in the trial is no more or less plausible than the assumptions made by the SDM.

Please note that the LBA model makes an important similar prediction to the SDM. When participants were interrupted by a click in the waiting period then the response to that click was faster when the EEG signal was more negative (Schurger et al., 2012). This is also predicted in our case. The more the signal has approached the threshold the shorter a motor reaction time would be if the accumulation processes for endogenous and exogenous movements share this common path. 

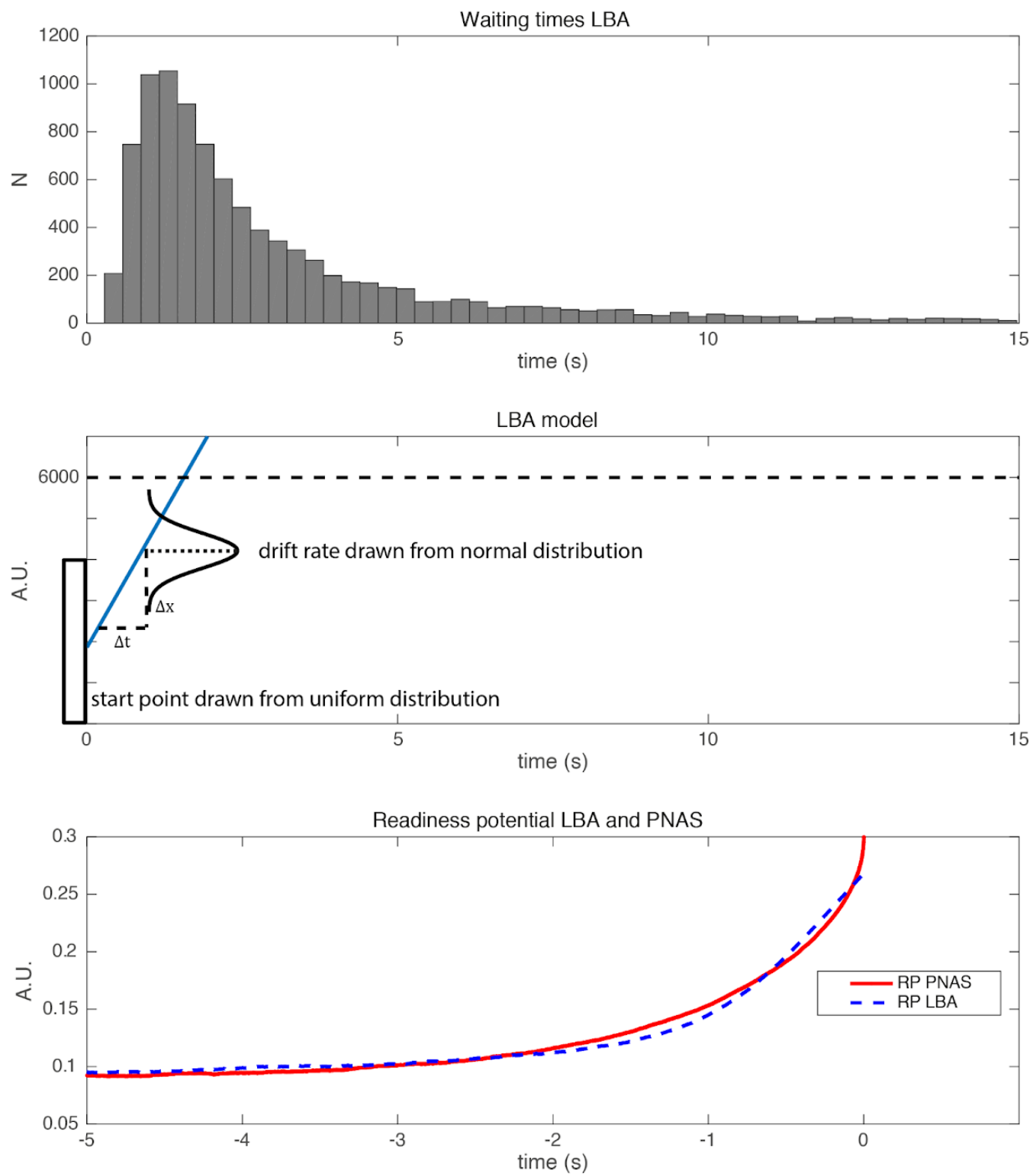

Figure 4: Simulation of a linear ballistic accumulator model (LBA). Top: Waiting times generated with the LBA. Middle: Schematic plot of the linear ballistic accumulator model. The starting position is drawn from a uniform distribution (between 0 and 4000). The drift rate is drawn from a normal distribution (mean $=1, \operatorname{std}=2$ ). The threshold is at 6000. Bottom: Readiness potentials generated with the LBA (blue) and the original SDM (red). Please note that the RP of the LBA is scaled in order to match the RP of the PNAS model (Schurger et al., 2012). In order to match empirical RPs both models involve additional scaling factors, which also ensure that the polarity of the time course is inverted to match the polarity of the empirical RP. 


\section{Confusion 4: What are the relative contributions of "noise" and "urgency"?}

In this section we are going to clarify the relative contributions of noise and urgency signals in the accumulation process. Note that Schurger et al. (2012) and many subsequent summaries frequently primarily focus on the fluctuations and largely ignore the role of the large initial ramp of the signal that is caused by the constant component. While they are indeed mentioned in the original manuscript, there are also other statements that focus solely on random fluctuations:

"One simple solution, given these instructions, is to apply the same accumulator-plus-threshold decision mechanism, but fed solely with internal physiological noise." (Schurger et al., 2012, p. E2905; underline added).

In a subsequent paper they say:

"[...] when actions are initiated spontaneously rather than in response to a sensory cue, the process of integration to bound is dominated by ongoing stochastic fluctuations in neural activity [...]" (Schurger et al., 2016, p. 78, underline added).

"In the case of spontaneous self-initiated movement there is no sensory evidence, so the process is dominated by internal noise." (Schurger et al., 2016, p. 77, underline added).

And even later as we have seen above:

"[...] the spontaneous action case is just an SDM driven by noise in the absence of evidence/reasons." (Schurger et al., 2021, p. 10, underline added).

Subsequently, many summaries of the findings ignore the role of the constant factor. For example, a subsequent version of the model, COINTOB, largely ignores this essential step as can be seen in their Fig. 1 (Brass et al., 2019). They write:

"[T]he threshold crossing is mainly determined by subthreshold neuronal noise [...]" (Brass et al., 2019, p. 256, underline added).

Here are more examples:

"A recent computational model [...] suggested instead that random fluctuations of a motor readiness signal could be sufficient to explain the initiation of voluntary actions[...]" (Ganos et al., 2015, p. 52, underline added).

"According to this model, the timing of the movement in the Libet experiment is determined by random threshold crossings in spontaneous fluctuations in neural activity. In particular, the model says that a decision when to move is determined by random 
threshold crossings only when it is not constrained by any evidence or reasons for action." (Schlosser, 2019, underline added).

Note that all these assertions would suggest that the urgency is 0 or close to 0 , which is not how it is actually modelled. As we have seen, if that were the case then it would take an implausible time to make the decision (cf. Fig. 2). In order to obtain a good fit between the model prediction and waiting times within their model the urgency seems to be necessary (Guevara Erra et al., 2019) and therefore moving the threshold close to 0 instead is not a solution. As we will see, quite the contrary is true: the urgency is the primary driving factor in threshold crossing.

If we look back to the perceptual decision making case the roles are quite clear. When the sensory information level is high then the accumulator primarily integrates the evidence throughout the trial (the component determined by $I$ in the model above). When the sensory information level is low, $I$ is set to 0 and the behavior is driven purely by the noise (plus potentially collapsing bounds). In the SDM the situation is different. $l$ is not set to 0 so there is a constant driving input. However, as we have seen most of the interpretation in the literature focuses on the role of the noise fluctuations.

So how could we quantify the relative role of these two processes? What is the contribution of either signal to reaching the threshold? In order to assess the contribution of the stochastic and the non-stochastic component we need to know how much of the total input to the accumulator has come from the noise and urgency components respectively. So we will sum up all the stepwise contributions of the noise component $\left(c \xi_{i} \sqrt{\Delta t}\right)$ and also of the urgency component $(I \Delta t)$ separately up to the point where the accumulator crosses the threshold, and ignoring the leak term (which operates at the level of the accumulated signal). The result of the simulation is shown in Figure 5 for both implementations of the model. For the first version of the SDM (Schurger et al., 2012) it can be seen that urgency dominates the input with a mean of 0.7 (averaged across 10000 trials) whereas the noise contributes on average 0 and in many trials even negative values (where the noise prevented the threshold from being crossed early) (Fig. 5, A). For the SDM implementation with pink noise as input (Schurger, 2018) the scenario is very similar. Urgency dominates the input with a mean of 0.33 (averaged across 10000 trials) and the noise contributes on average 0 (Fig. 5, B). The smaller values for the second model (Schurger, 2018) are due to a lower threshold compared to the first model (Schurger et al., 2012) (0.298 vs. 0.1256).

The contribution of urgency is directly dependent on the length of the trial because it is a constant input into the accumulator. In each time step $I \Delta t$ is added. Therefore the contribution of urgency is always positive. Overall, the noise contribution is negatively correlated with the waiting time. In short 
trials the noise contribution is positive, because the noise is necessary to push the total signal over the threshold. Long trials on the other hand are the result of zero or a negative contribution of noise, otherwise the threshold would have been crossed earlier. The longer the trial the more negative is the accumulated noise contribution.
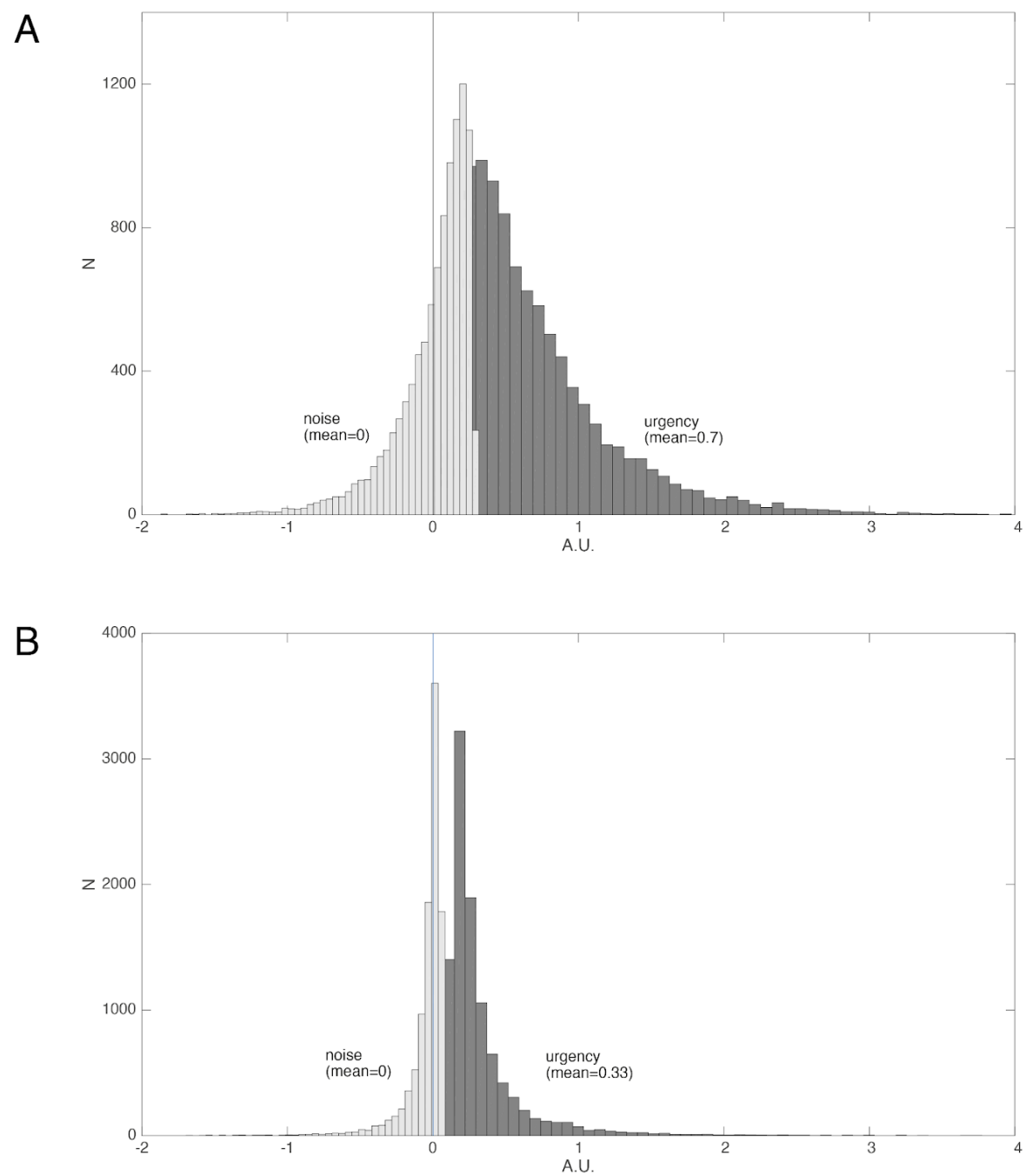

Figure 5: Total accumulated absolute contribution of urgency (dark gray, right histogram) and noise (light gray, left histogram). The total contribution of urgency is always positive whereas the contribution of noise is in many trials even negative. Urgency dominates the overall input into the accumulator. A PNAS version of the SDM (Schurger et al., 2012). B The finding is very similar for subsequent extended SDM (same scaling)(Schurger, 2018).

However, one might consider our emphasis on the importance of urgency a distraction. Why is this so important? There are important reasons to highlight the role of urgency: in the model the urgency characterizes a fully deterministic component of the decision that is fixed once the trial has begun. 
The data by Murakami et al. (2014) also suggest that the information about when the decision is made is already there early in the trial. Furthermore, the LBA model where all the decisions are made at the beginning of the trial also predicts the RP and the distribution of waiting times (Brown \& Heathcote, 2008). We will see below that these facts might change the interpretation about whether the decision is made early or late. As we will explore in the next section, there is a common view that the deterministic component is simply a preparatory stage, that brings the signal within reach of the threshold and then subsequently fluctuations take over. We will see that this is also not the case.

\section{Confusion 5: Does the "urgency" (constant) term first bring the system into a dynamic range where random fluctuations take control?}

As we have seen above there is another important aspect of the model, that noise- and urgency-related processes are interpreted as constituting separable and sequential stages (for examples see above). The idea there would be that the constant signal initially drives a "stochastic exponential transition period" (Schurger et al., 2012, p. E2906) that first brings the accumulated signal into an operating range, and subsequently the fluctuations determine when the signal crosses the threshold (Schurger et al., 2012, p. E2906). Here are a few examples of this point:

\footnotetext{
"After a stochastic exponential transition period [...], the leaky accumulator generates noisy trajectories whose threshold crossings determine movement times." (Schurger et al., 2012, p. E2906);

"In our model this solution amounts to simply shifting premotor activation up closer to the threshold for initiation of the instructed movement and waiting for a random threshold-crossing event." (Schurger et al., 2012, p. E2905);

"According to their stochastic decision model, the decision process, given Libet's instructions, amounts to simply shifting premotor activation up closer to the threshold for initiation of the movement and waiting for a random threshold-crossing fluctuation in RP." (Bayne \& Pacherie, 2015, p. 224).
}

Considering these statements we should expect two effects: First, the accumulator is moved closer to the threshold without any (or only few) decisions being made. Second, from this plateau the system waits for a random threshold-crossing event.

Let us consider the time point five seconds into the trial where the accumulator has on average reached around $90 \%$ of its asymptote (see Fig. 6, middle). One might assume that hardly any 
decisions have been made by this point, but quite the opposite is the case. In $52.7 \%$ of the trials the threshold is crossed and a decision is made earlier than $5 \mathrm{~s}$. For the trials with long waiting times it can even be observed that the signal fluctuates strongly and sometimes even reaches negative values after it was first closer to the threshold (see the orange and yellow curves on the right of Fig. 6, bottom). Therefore, the "move signal closer to threshold" process can even happen multiple times in slow trials. Thus, the verbal description and interpretation of noise and urgency does not capture the model behavior appropriately. During the whole time course of a trial both urgency and noise contribute to the current state of the accumulator. It's a concert of the two, both contributing to the process, similar to accumulator models in perceptual decision making. Please note, that above arguments also hold if time points earlier than $5 \mathrm{~s}$ are considered as reaching a lenient interpretation of a plateau. We observe many early decisions and the SDM signals fluctuate strongly.

Please note, in the extended version of the SDM with pink instead of white noise as input (Schurger, 2018) the logic of the two stages is even more problematic. There, urgency can drive the SDM across the threshold alone, i.e. without the noise, and thus two sequential stages are not necessary anymore. Here is the proof: we can calculate $x_{i}$ at the asymptote, i.e. when $\Delta x_{i}$ is zero. We also consider the case without noise.

$$
\begin{gathered}
\Delta x_{i}=I \Delta t+c \xi_{i} \sqrt{\Delta t}-k x_{i} \Delta t \\
0=I \Delta t+0-k x_{i} \Delta t \\
k x_{i} \Delta t=I \Delta t \\
x_{i}=\frac{I}{k}
\end{gathered}
$$

With the reported parameters $(I=0.1, k=0.6$ and threshold $=0.1256)$ the SDM would converge to $0.1 / 0.6=0.167$ based on urgency alone and without noise. The threshold in this model is at 0.1256 so the threshold would be crossed without any noise in the model. 

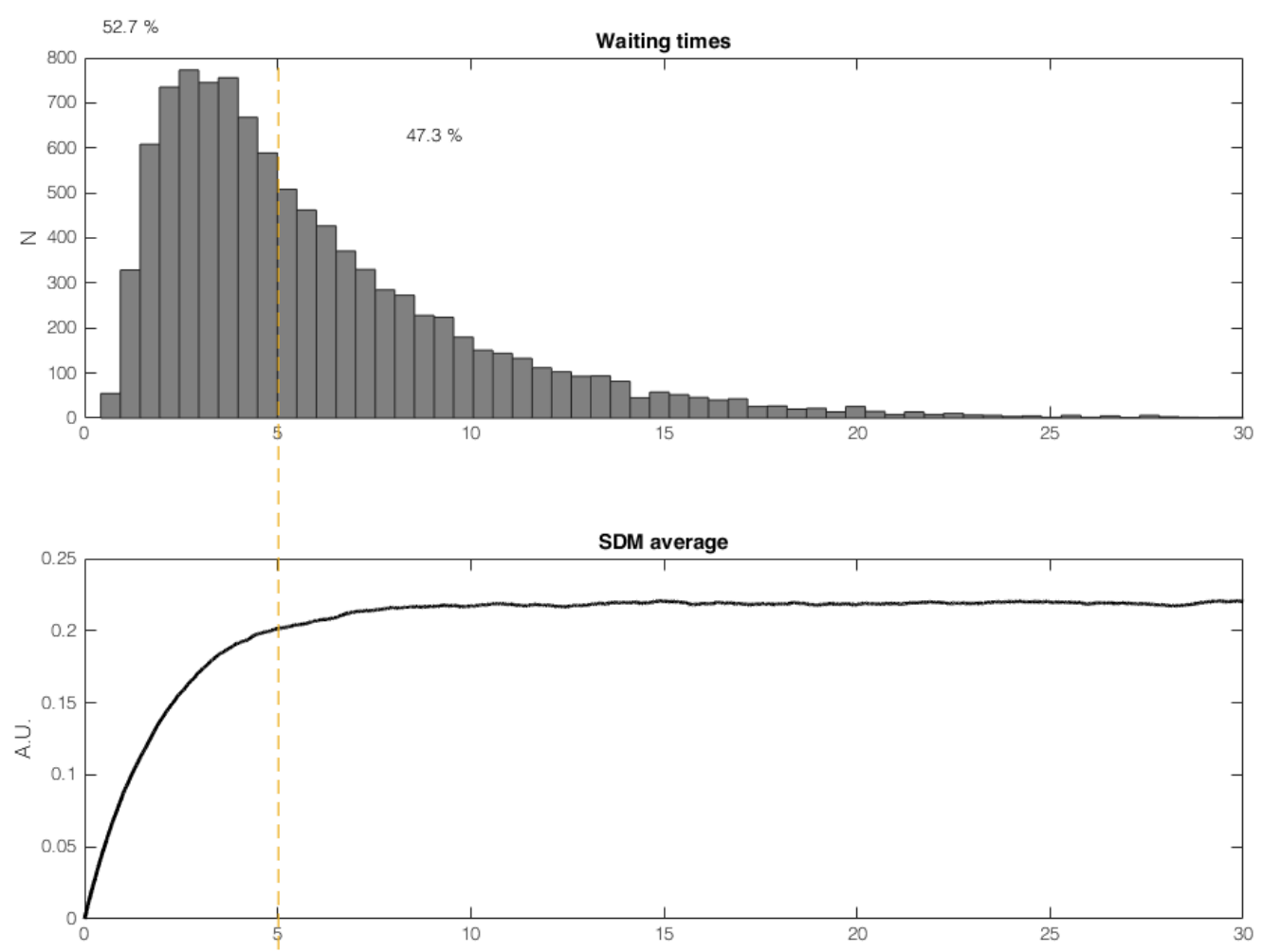

Figure 6: Result of 10000 trials of the SDM calculated with the parameters reported in Schurger et al. (2012). Top: Histogram of waiting times. Middle: Average SDM output of 10000 trials. The average SDM converges after around $5 \mathrm{~s}$. In the averaged signal, the noise across trials cancels out and the result is similar to a SDM with only urgency and no noise as input (Fig. 1). Bottom: 10 sample trials of the SDM (truncated after crossing the threshold, dashed line). More than half of the trials (52.7\%, left side of orange line) cross the threshold before $5 \mathrm{~s}$. Trials with long waiting times don't stay close to the asymptote but fluctuate strongly. Both, trials with a waiting time faster than $5 \mathrm{~s}$ and trials with a very slow waiting time, don't show the proposed behavior that the signal is moved closer to the threshold and that then some noise causes a threshold crossing. Urgency and noise both continuously influence the SDM signal, while urgency is only driving the signal up, noise is driving the signal up and down. 


\section{Confusion 6: Is any "evidence" involved in the model?}

In perceptual decision making, "evidence" refers to one variable having information about another, such as a perceptual representation having evidence about an external stimulus. This is the reason it is called "evidence" and not simply "a signal in MT" or "bias". In contrast, in the SDM the urgency describes an intrinsic signal that is not evidence, but a signal that adjusts the baseline to a range where accumulated noise fluctuations can take control. In line with this, the authors of an animal study on endogenous movement decisions that they consider to reflect an accumulation process (Murakami et al., 2014), say that their task involves "no evidence per se" (p. 1580).

The SDM seems first to be very clear about the urgency being dedicated to the purpose of bringing the signal into the threshold range, not about encoding say some internal motivation state. However, there seems to be some confusion in the literature about whether one of the signals (urgency or noise) might reflect evidence in the SDM after all. Already an early review paper interpreted the original study as

"showing that bounded-integration processes, which involve the accumulation of noisy evidence until a decision threshold is reached, offer a coherent and plausible explanation for the apparent pre-movement build-up of neuronal activity." (Schurger et al., 2016, p. 77, underline added).

Brass, Fürstenberg \& Mele (2019) interpret the original paper on the SDM as a solution that:

"treat[s] stochastic noise in the motor system as evidence for the accumulation process" (Brass et al., 2019, p. 256, underline added).

They then continue:

"In contrast to perceptual decision making, however, the accumulation of evidence [in the SDM] is not based on perceptual information but on internal information and stochastic neural activity. "(p. 259 , underline added).

And then:

"These models assume that decision time in the Libet task is based on a process of accumulation of evidence to a threshold, just like in other decision-tasks. Because the decision is not based on perceptual or other external evidence, this accumulation of evidence might operate primarily on stochastic neural fluctuations in the motor system" (p. 257, underline added). 
And finally:

"This means that the RP and the LRP do not reflect a ballistic process that necessarily leads to action but rather a gathering of evidence." (p. 259, underline added).

Thus, it appears that the noise seems to play a role of evidence, and that there is some additional signal involved, here termed "internal information". Also in other papers there is some ambiguity as to the respective roles of the variable factor (i.e. the noise fluctuations) and the constant factor (evidence/urgency):

"Schurger et al. propose that the motor system constantly undergoes random fluctuations of RPs and that this random premotor activity is used as a substitute for actual evidence." (Pacherie, 2014, p. 36).

Of course it is possible to go beyond the original formulation of the SDM and re-consider the urgency signal as having some computational function dedicated to representing decision-relevant internal states (such as motivation or impulsivity). But when considering one variable having evidence about another one would want it to fulfill some additional requirements. For example, the evidence should be able to "stand in" as a proxy of what it is representing. To illustrate this, we may turn to Brass et al. (2019), who note that it could indeed be sensible to assume that some latent internal signals could influence the buildup of the urgency when no external information is available. In such cases, the level of urgency could be somewhat constrained by these causally influencing factors, but we would not necessarily see urgency as having a function of reliably tracking such variables and serving as a stand-in (i.e. "evidence") for those. Not every causal influence can be considered as evidence. Please further note that if the urgency indeed played a role of collecting evidence it would also have needed to receive much more attention as an integral part of the movement decision, and not be largely ignored as we have seen above.

\section{Confusion 7: Is the decision early or late?}

As mentioned above, a classical interpretation of the readiness potential is that it reflects a post-decisional stage of processing after an unconscious decision to act has been made (Schurger et al., 2012). First the brain makes an early decision, then a process (of which the RP is an indicator) is triggered that prepares the movement. Many consider this to be counterintuitive because of the long temporal delay of a few hundred ms between the brain's unconscious decision and the time when a participant consciously believes to be "making the decision now" (Libet et al., 1983). An important reason for the interest in the stochastic decision model is that it seems to remove this counterintuitive time delay. It implies instead that the decision to act occurs late, that is when the 
accumulated signal crosses the threshold for action (Brass et al., 2019; Schurger et al., 2012), which is much closer to the subjective time of decision. Everything happening before that is just some random background activity in the brain. In this view the readiness potential originates already at a pre-decisional stage and is an artefact of averaging stochastic signals.

Just as a reminder, "decision" here can mean two things: (a) the participant's conscious experience of making a decision, and (b) some (potentially unconscious) brain event, quasi a "neural decision" ${ }^{1}$, that somehow sets the brain on the track for executing the movement (these two events may or may not coincide in time). Because the discussion of the SDM has focused on the threshold crossing, which is a property of the neural system, we will focus on neural decisions and set the problem of subjective decisions aside. And in order to avoid a too extended general discussion of the role of randomness and (in)determinism in biological systems, we will base our discussion on properties of the computational models. The question we want to discuss here is whether the SDM really provides sufficient evidence to support a late decision model. We would like to suggest that the interpretation of the nature of the fluctuations in the SDM is vital when interpreting the decision as early or late.

In the papers on the SDM the fluctuations are described as "internal physiological noise" or "random fluctuations" (Schurger et al., 2012, p. E2905 and E2904). This could mean different things, so an important distinction is required. (a) Either this could mean that they are "objectively random" in the sense of the randomness being an irreducible part of the world that would not be predictable in more detail however much we learn about the world. An extreme version of this were if fluctuations were guided by quantum processes (analogous to e.g. radioactive decay) ${ }^{2}$. Due to their indeterminism such fluctuations could not be predicted. Whatever one might find out about the states of the world, there is an intrinsic indeterministic rest. (b) On the other hand the randomness could refer to "epistemic randomness", where a signal would appear to have quasi-random properties, but this would reflect the fact that some properties of the process are not known (e.g. due to insufficient data or to not understanding the algorithm, say as in the case of a deterministic random number generator). The process could also be unpredictable despite being deterministic (as for example in deterministic chaos). The randomness might also constitute a mixture of (a) and (b), so we might uncover more latent determinants as research progresses, but there still remains an irreducible rest.

Now let us see whether the interpretation of the nature of the processes leading up to the threshold influences at which time we would consider the decision to happen. In order to cleanse our thinking

\footnotetext{
${ }^{1}$ We use the term "decision" here without further discussion, but consider a clarification of what could constitute a decision without making reference to the subjective experience a major challenge in this field. From a neural level of resolution, brain processes can be described as trajectories in a high-dimensional state-space. It is unclear what would constitute a decision along such a trajectory. If one considers phase transitions or bifurcations as decisions, as criteria then these would be ubiquitous.

${ }^{2}$ At least in indeterministic interpretations of quantum mechanics.
} 
of the a-priori assumptions we have with accumulator models, let us consider a physical analogy, a Rube Golderg-style simple chain reaction model of a ball rolling down a slope towards a row of dominos (Fig. 7). The dominos fall over one by one and then ring a bell. In each trial the ball is set off with a slightly different speed and we measure the time until the bell is rung. Note that when the ball knocks over the first domino stone a nonlinearity is reached, such that a different process is triggered. If the ball for some reason were prevented from reaching that stage, say it were to mysteriously move uphill again, then the chain reaction in the dominos would not be triggered. Thus, knocking over the first domino is similar to passing a nonlinear threshold (analogous to $\beta$ ).

We will consider four different cases: (1) In the first case (Fig. 7, top) a ball rolls down a constant smooth slope, knocks over the first domino, which triggers a predictable chain of events until the last ball rings the bell. Everything in this model is perfectly determined at the beginning of the trial when the ball is set off with a certain speed. So when is the decision made that determines when the bell is rung? (2) In the second case (Fig. 7, middle) everything is the same, with the exception that the surface is now rough. Let's assume that the rough surface causes the ball to bump back and forth on the way, thus adding a random component to the time it takes to pass the ramp. Let's further assume that the effect of these bumps is indeterministic in principle, so that however precise we can measure the ball and the surface there would still remain a rest of indeterminacy. When is the decision made here? (3) Now let's consider a third case, similar to (2) but now the effect of the bumps is perfectly deterministic. When the ball is let go with a certain speed and the same rough surface the run time is always the same. When is the decision made in this deterministic case? (4) Let's also consider a variant on (3). Now in every trial we use a different rough slope (Fig. 7, middle and bottom). Each of these slopes is perfectly deterministic, but we don't know which one is chosen on a given trial. When is the decision made here?

Cases 1 and 3 are clear determinism. Case 2 is indeterminism. And case 4 is concealed determinism that appears as indeterminism because a latent variable (which slope is chosen) is not known. The rolling down the slope is an analogy to the drift phase and the domino stones are an analogy for the motor execution stage triggered after passing the threshold. If all the relevant causal factors are established (as in cases 1, 3 and 4), isn't the outcome of the decision then pre-determined and it can thus be considered already made? 


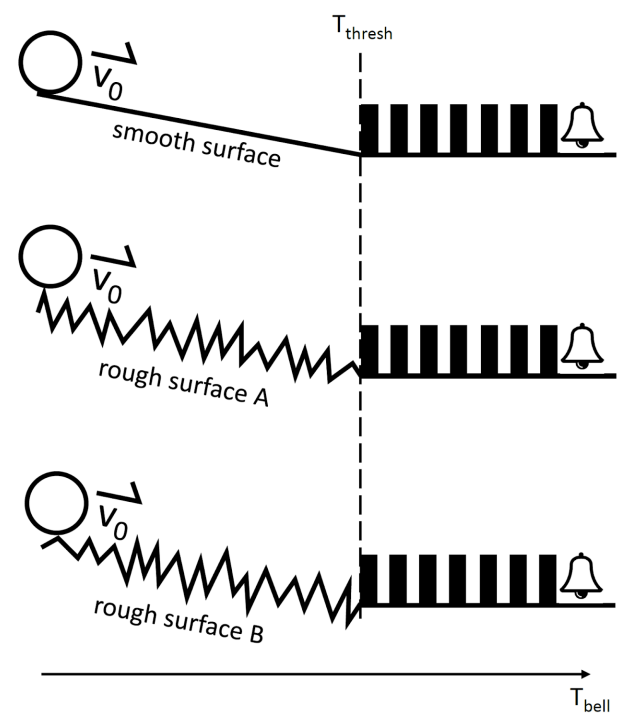

Figure 7: Simple causal chains inspired by Rube Goldberg to illustrate the models. Top: A ball is set off on an even slope with variable speed $v_{0}$ that is determined at the beginning of the trial from a Gaussian random distribution. The slope is chosen to exactly balance the friction and keep the ball at a constant speed without acceleration or deceleration. At the end of the slope at time $T_{\text {thresh }}$ the ball triggers a chain of dominos that runs deterministically through and then sounds a bell at time $T_{\text {bell }}$. Note that if the ball (for some reason) were to be stopped from reaching the dominos they would not be triggered, so the passing of the threshold can be considered a nonlinear event. When would the decision be made when the ball reaches the bell? There is only one free variable: the speed with which the ball is initially set off. So is the decision made at the beginning of the trial? Middle: Now the same causal chain is set off but with an added rough surface. Now the time that is needed to pass the rough slope influences the time until the bell is rung. But is this process deterministic? That depends on the nature of the rough surface. One type of surface would potentially result in slightly different bumps on every trial and thus add some irreducible variability to the run time. A different type of surface might still potentially yield reproducible run times. Bottom: Now, let's stick with the reproducible version and exchange the rough slope on a trial-by-trial fashion, each time with a different reproducible slope. Let's assume we don't know which slope has been picked on a given trial. When is the decision about the run time made? Given that following the release of the ball everything is fully deterministic, one plausible interpretation would be to say that the decision is made at the beginning of the trial, despite appearing to depend on random processes on the way. Even though the model only reproduces some properties of the accumulator models (for example there is no leak) it can help form our intuitions about which events might count as decision points. Without an in-depth understanding of the nature of the noise and without an ability to measure it, it is impossible to say whether a process is deterministic or not. And whether the decision is early or late will presumably depend on the answer to this question.

\section{Confusion 8: Does the model explain the lag between awareness and decision?}

The time when subjects experience wanting to act is referred to as W-time (Libet et al., 1983). To avoid confusion we will call this the awareness time $\left(T_{\text {awareness }}\right)$. This awareness time comes later than the onset of the readiness potential, which could be taken to mean that the brain unconsciously makes a decision a few hundred ms before the experience of wanting to move. Schurger (2018) now 
provides a solution to this seemingly implausible long gap between the brain's decision and the conscious decision. For this he introduces a second threshold $\left(\beta_{\text {awareness }}\right)$, slightly lower than the main movement threshold $\beta$ (in their matlab code it is set at $96.67 \%$ of the main threshold). The idea is that crossing this first threshold "informs" the awareness time. The idea is that the lag between the first and the second threshold is one of several inputs that feed into the estimation of awareness time.

However, there is a problem with this explanation. That is because the accumulated signal can cross the awareness threshold but then proceed to decrease again without triggering a movement. This is not even that rare. When taking the model at face value, before crossing the threshold $\beta$ the slightly lower threshold $\beta_{\text {awareness }}$ can even be crossed multiple times due to the random nature of the accumulation of fluctuations. That is because due to its random waxing and waning the accumulator signal can also decrease and in theory even reach 0 again. This leads to high variability in threshold-crossing latencies, thus making it difficult to explain awareness time without additional assumptions. Currently, there is no justification why the last (rather than the first) threshold crossing of $\beta_{\text {awareness }}$ should determine awareness time. In their MATLAB code Schurger (2018) picked the last (of potentially many) threshold-crossings as the indicator for awareness time.

To demonstrate this, we plotted the timing of the first versus the last crossing of $\beta_{\text {awareness }}$ (Schurger, 2018; Schurger et al., 2012). The results can be seen in Figure 8 . In many trials $(73.24 \%$ for the PNAS version of SDM and still 5.95\% for the eNeuro version of the SDM) the threshold $\beta_{\text {awareness }}$ is crossed at least twice. More importantly the time lag between the first and the last crossing can be higher than $20 \mathrm{~s}$ (for both versions of the model), which is an implausible lag. Also, there is no reason why the last but not the first threshold crossing informs awareness time. Please note that the LBA is in line with the prediction of a correlation between T and awareness time (Schurger, 2018, Figure 5 and Figure 7). 
A
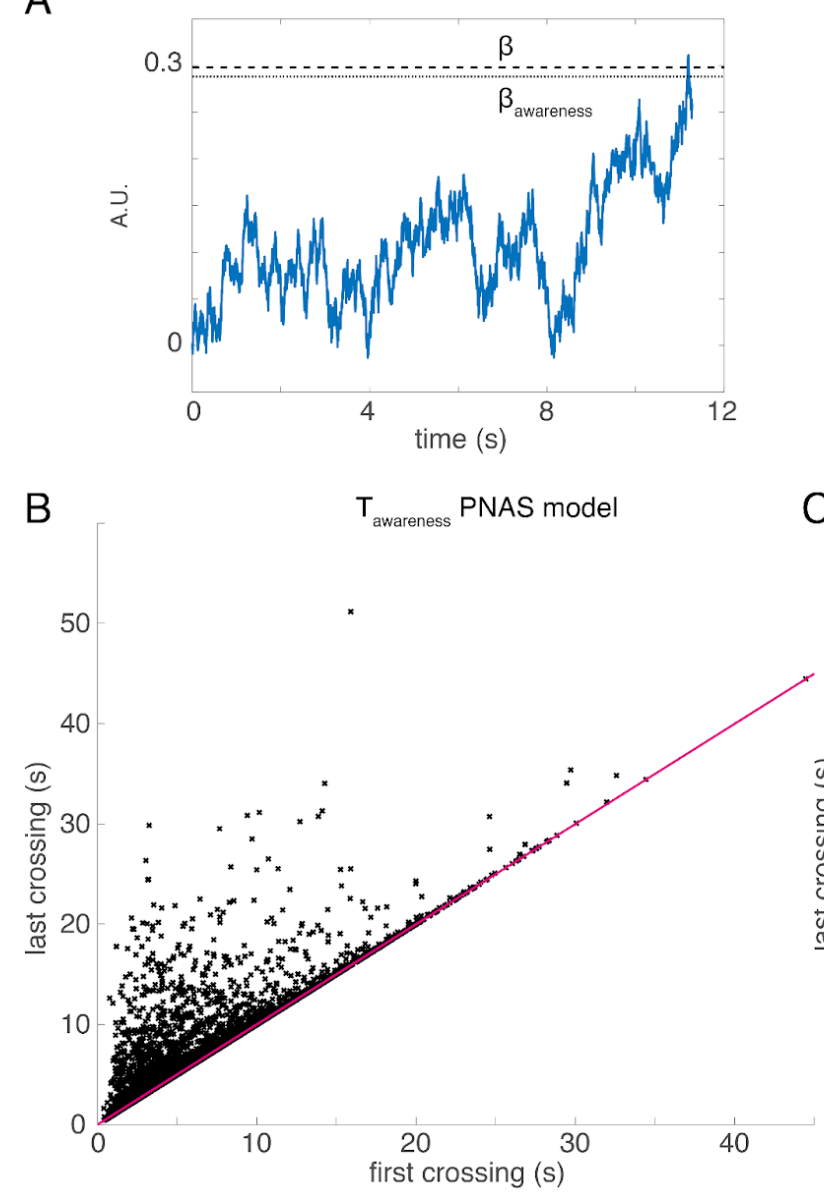

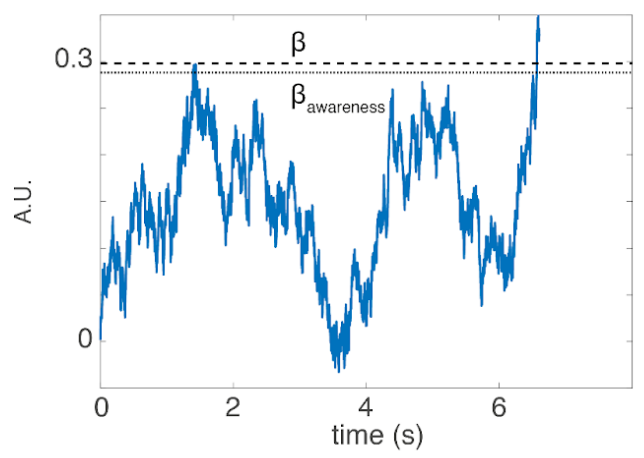

C

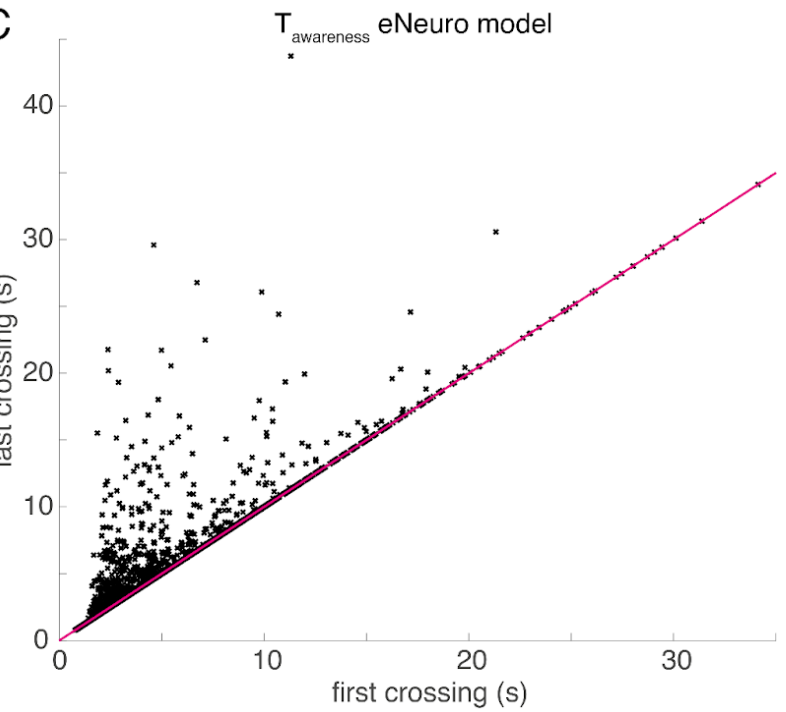

Figure 8: A: Two single trials of the PNAS version of the SDM (Schurger et al., 2012). Left: After about $11 \mathrm{~s}\left(\beta_{\text {awareness }}\right)$ the threshold to awareness is crossed and briefly after the threshold for triggering a movement $(\beta)$ is crossed (please note that the overall reaction time would additionally include a motor response latency, which is ignored in these models). Right: Here the awareness threshold is crossed relatively early at about $2 \mathrm{~s}$ but the motor threshold is not crossed immediately after that. Due to the random nature of the accumulation the signal even moves down towards zero before it moves up again and then crosses both thresholds at $\beta_{\text {awareness }}$ and then $\beta$ after around $7 \mathrm{~s}$. B: Scatterplot of the first and last crossing of $\beta_{\text {awareness }}$ for the PNAS version of the SDM. In $73.24 \%$ of the trials the threshold is crossed at least twice. The diagonal (red) is the identity line. It shows trials where the threshold is only crossed once (i.e. first and last crossing is identical). All points above the identity line are cases where the final threshold crossing is later than the first. The delays can extend to the $10 \mathrm{~s}$ of seconds. C: Scatter plot of the first and last crossing of the threshold $\beta_{\text {awareness }}$ for the eNeuro version of the SDM (Schurger, 2018). Because the relative influence of urgency is stronger in this version of the SDM the number of multiple crossings is reduced. Nonetheless, still in $5.95 \%$ of the trials the awareness threshold is crossed at least twice.

\section{Summary and outlook}

The aim of this paper was to assess the level of evidence for the SDM and to address certain confusions that have arisen in the literature. First, we highlighted that there is no evidence based on direct measurements for the role of stochastic fluctuations and that the analyses are based on macroscopic signals averaged across many trials. This does not rule out the SDM as a model, but it 
clarifies how far removed the measured data are from the hypothetical single-trial fluctuation time courses. Second, we found that the support for the SDM from animal studies is limited and partially even in contradiction to the SDM. Third, we showed that a deterministic model where the parameters are fixed at the beginning of the trial makes very similar predictions to the SDM, including for the interrupted version of the Libet task. Thus, fluctuations may not be needed. Fourth, we found that the literature has not adequately characterized the deterministic component of the SDM, the urgency signal, that accounts for the vast majority of signal input towards the threshold. The fluctuations in contrast have a much smaller (and sometimes even negative) contribution. Fifth, there is a confusion regarding the link between perceptual decision making and spontaneous movements. We have shown that the SDM is not just a special case of perceptual decision making, just without the evidence. That is because evidence, urgency and noise play very different roles in the SDM and in perceptual decision making. It is misleading to consider the urgency signals in SDM as a form of evidence or information, at least in the current formulation. Sixth, we found that a key aim of the SDM to provide a predecisional account of the RP cannot really be addressed by the model because this hinges critically on the nature of the noise fluctuations as being "objectively random" versus "epistemically random". This of course will be very difficult or even impossible to decide empirically, but at least the case is far from closed. Finally, we found that due to the multiple crossings of the hypothetical awareness threshold, the SDM would predict a variety of time lags between the conscious decision and a hypothetical neural decision, raising the question of how this can explain awareness time.

There are some other questions that need to be addressed: Where is the ramp? Is there any insight into the neural mechanisms underlying the large initial urgency-based ramp at the beginning of the trial where the accumulator signal moves closer to the threshold? If this process involves the same neural mechanism as the readiness potential (i.e. it reflects the signal in the accumulator), then one would expect to see a very stereotypical ramp at the beginning of the trial that should be much stronger than the RP and that exactly matches its topography.

Could the ramp not be the buildup of the intention? The fact that the urgency signal plays such an important role in bringing about the decision could point to a re-interpretation of the components of the SDM model. Couldn't one interpret the urgency signal as the largely deterministic and gradual buildup of the intention to move? And the smaller effect of randomness would just reflect say slight imperfections in biological processes from trial to trial or the variability in motivation with which the participants start a trial? Random variability from trial to trial is observed in just about any task (from threshold perception to motor performance) without anyone claiming that this randomness is the key aspect of the process. 
It could also be useful to extend the scope from thinking about spontaneous movements in general to what happens specifically in spontaneous movement experiments. These lab experiments impose constraints that are not present in real-world free-ranging actions. For example, there is an explicit or implicit affordance to move within a reasonable time-frame (e.g. to not wait too long) and at the same time avoid being predictable or rhythmic. Already the earliest paper on readiness potentials stated: "The participant was required to perform the movement not rhythmically, but in irregular intervals" (Kornhuber \& Deecke, 1965, p. 1 , our translation). In the study by Schurger et al. (2012), the instructions are to "[...] try not to decide or plan in advance when to press the button, but to make the event as spontaneous and capricious as possible." (Schurger et al., 2012, p. E2911). What if the participant is thinking: "Oh dear, am I spontaneous or capricious enough?", and if so what would they do? In the classic Libet study the participants are required to "[...] let the urge to act appear on its own at any time without any preplanning or concentration on when to act" (Libet et al., 1983, p. 625). One might wonder what participants were thinking if they didn't experience such a mysterious "urge". Would they have just waited for the whole duration of the experiment and then finished the experiment by saying: "Sorry, but I never felt an urge to move"? The key point here is that the preparation of these movements might have involved a vast array of cognitive processes, conscious or unconscious. These could include (among others) mental time keeping and time-based prospective memory (McDaniels \& Einstein, 2000), inhibition of behavioral impulses to move immediately (Noorani \& Carpenter, 2017), or generation of random behavior sequences (Nickerson, 2002). Sticking with the latter, obviously, in order to be random and capricious one could in principle use a random time interval generator based on the accumulation of fluctuations. But why generate or use a long series of random numbers, if all you have to do is generate one single random number at the beginning of the trial (as e.g. in the LBA)?

In a recent review paper the authors summarize the evidence for the SDM:

"Why should there be such a long and highly variable lag, of up to one second or more, between the decision to initiate movement and movement onset? Why has the RP not proven to be a very reliable real-time predictor of movement onset? And why are subjective reports of the (conscious) decision time so late relative to the supposed 'onset' of the RP? These puzzling questions are not at all puzzling from a late-decision perspective, so the onus should be on proponents of the early-decision account to explain why the more parsimonious late-decision view is false." (Schurger et al., 2021, p. $5)$.

These questions might have simple answers. For example, the delay after the brain's early decision to move until the movement occurs could simply reflect a delay that is decided upon already at the 
beginning of the trial and that is introduced to comply with the instructions of the task, similar to the LBA. That would also predict why the RP onset is not a reliable predictor of the movement onset. Furthermore, the LBA model is more parsimonious than the SDM because it involves fewer variables (i.e. it does without the unmeasured and thus hypothetical fluctuation time series). The only point that might remain, is that the SDM seems to provide an explanation for why we subjectively believe to be making the decision late, rather than early. However, such discrepancies between subjective experience and latent brain processes are not unusual (e.g. van Gaal \& Lamme, 2012), and we do not consider it a desirable aim of cognitive neuroscience to make our scientific findings fit to our naive folk-psychological models of how our mind works for the pure sake of it. We would like to clarify that we do not want to argue that the verdict is already in for an early decision model (as in the LBA), or that the SDM can be finally ruled out based on the evidence. At the current state of evidence it is impossible to use the SDM to argue for pre- or postdecisional models of the RP. We believe that the empirical support for the model is currently very weak, that there are viable alternatives and that also several key conceptual issues still need clarification.

\section{Acknowledgements}

This work was funded by the Excellence Initiative of the German Federal Ministry of Education (Excellence Cluster Science of intelligence), the BMBF (through the Max Planck School of Cognition), the DFG (GRK 2386 "Extrospection"), and a joint grant by the John Templeton Foundation and the Fetzer Institute. We would like to thank Matthias Schultze-Kraft, Thomas Goschke, Marcel Brass and Michael Pauen for valuable discussions on the topic.

\section{References}

Bayne, T., \& Pacherie, E. (2015). Consciousness and Agency. In J. Clausen \& N. Levy (Hrsg.), Handbook of Neuroethics (S. 211-230). Springer Netherlands. https://doi.org/10.1007/978-94-007-4707-4_117

Bode, S., Bogler, C., \& Haynes, J. D. (2013). Similar neural mechanisms for perceptual guesses and free decisions. Neuroimage, 65, 456-465.

Brass, M., Furstenberg, A., \& Mele, A. R. (2019). Why neuroscience does not disprove free will. Neuroscience \& Biobehavioral Reviews, 102, 251-263. https://doi.org/10.1016/j.neubiorev.2019.04.024 
Brown, S. D., \& Heathcote, A. (2008). The simplest complete model of choice response time: Linear ballistic accumulation. Cognitive Psychology, 57(3), 153-178. https://doi.org/10.1016/j.cogpsych.2007.12.002

Eccles, J. C. (1985). Mental summation: The timing of voluntary intentions by cortical activity. Behavioral and Brain Sciences, 8(4), 542-543. https://doi.org/10.1017/S0140525X00044952

Friston, K. J., Harrison, L., \& Penny, W. (2003). Dynamic causal modelling. Neurolmage, 19(4), 1273-1302. https://doi.org/10.1016/s1053-8119(03)00202-7

Ganos, C., Asmuss, L., Bongert, J., Brandt, V., Münchau, A., \& Haggard, P. (2015). Volitional action as perceptual detection: Predictors of conscious intention in adolescents with tic disorders. Cortex; a Journal Devoted to the Study of the Nervous System and Behavior, 64, 47-54. https://doi.org/10.1016/j.cortex.2014.09.016

Guevara Erra, R., Arbotto, M., \& Schurger, A. (2019). An integration-to-bound model of decision-making that accounts for the spectral properties of neural data. Scientific Reports, 9(1), 8365. https://doi.org/10.1038/s41598-019-44197-0

Hawkins, G. E., Forstmann, B. U., Wagenmakers, E.-J., Ratcliff, R., \& Brown, S. D. (2015). Revisiting the evidence for collapsing boundaries and urgency signals in perceptual decision-making. The Journal of Neuroscience: The Official Journal of the Society for Neuroscience, 35(6), 2476-2484. https://doi.org/10.1523/JNEUROSCI.2410-14.2015

Kornhuber, H. H., \& Deecke, L. (1965). HIRNPOTENTIALAENDERUNGEN BEI WILLKUERBEWEGUNGEN UND PASSIVEN BEWEGUNGEN DES MENSCHEN: BEREITSCHAFTSPOTENTIAL UND REAFFERENTE POTENTIALE [CHANGES IN THE BRAIN POTENTIAL IN VOLUNTARY MOVEMENTS AND PASSIVE MOVEMENTS IN MAN: READINESS POTENTIAL AND REAFFERENT POTENTIALS]. Pflugers Archiv Fur Die Gesamte Physiologie Des Menschen Und Der Tiere, 284, 1-17.

Latimer, K. W., Yates, J. L., Meister, M. L. R., Huk, A. C., \& Pillow, J. W. (2015). Single-trial spike trains in parietal cortex reveal discrete steps during decision-making. Science, 349(6244), 184-187. https://doi.org/10.1126/science.aaa4056

Libet, B., Gleason, C. A., Wright, E. W., \& Pearl, D. K. (1983). Time of conscious intention to act in 
relation to onset of cerebral activity (readiness-potential). The unconscious initiation of a freely voluntary act. Brain: A Journal of Neurology, 106 (Pt 3), 623-642. https://doi.org/10.1093/brain/106.3.623

Libet, Benjamin. (1985). Unconscious cerebral initiative and the role of conscious will in voluntary action. Behavioral and Brain Sciences, 8(4), 529-539. https://doi.org/10.1017/S0140525X00044903

McDaniels, M. A., \& Einstein, G. O. (2000). Strategic and automatic processes in prospective memory retrieval: A multiprocess framework. Applied Cognitive Psychology, 14(Speclssue), S127-S144. https://doi.org/10.1002/acp.775

Murakami, M., Vicente, M. I., Costa, G. M., \& Mainen, Z. F. (2014). Neural antecedents of self-initiated actions in secondary motor cortex. Nature Neuroscience, 17(11), 1574-1582. https://doi.org/10.1038/nn.3826

Nickerson, R. S. (2002). The production and perception of randomness. Psychological Review, 109(2), 330-357. https://doi.org/10.1037/0033-295x.109.2.330

Noorani, I., \& Carpenter, R. H. S. (2017). Not moving: The fundamental but neglected motor function. Philosophical Transactions of the Royal Society B: Biological Sciences, 372(1718), 20160190. https://doi.org/10.1098/rstb.2016.0190

Pacherie, E. (2014). Can Conscious Agency Be Saved? Topoi, 33(1), 33-45. https://doi.org/10.1007/s11245-013-9187-6

Ratcliff, R. (1978). A theory of memory retrieval. Psychological Review, 85(2), 59-108. https://doi.org/10.1037/0033-295X.85.2.59

Ringo, J. L. (1985). Timing volition: Questions of what and when about W. Behavioral and Brain Sciences, 8(4), 550-551. https://doi.org/10.1017/S0140525X00045052

Schlosser, M. E. (2019). Agency. In Stanford Encyclopedia of Philosophy (Winter 2019 Edition). https://plato.stanford.edu/archives/win2019/entries/agency/

Schultze-Kraft, M., Birman, D., Rusconi, M., Allefeld, C., Gorgen, K., Dahne, S., Blankertz, B., \& Haynes, J. D. (2016). The point of no return in vetoing self-initiated movements. Proc. Natl. Acad. Sci. 
U.S.A., 113(4), 1080-1085.

Schurger, A. (2018). Specific Relationship between the Shape of the Readiness Potential, Subjective Decision Time, and Waiting Time Predicted by an Accumulator Model with Temporally Autocorrelated Input Noise. ENeuro, 5(1). https://doi.org/10.1523/ENEURO.0302-17.2018 Schurger, A., Hu, P. „Ben“, Pak, J., \& Roskies, A. L. (2021). What Is the Readiness Potential? Trends in Cognitive Sciences. https://doi.org/10.1016/j.tics.2021.04.001

Schurger, A., Mylopoulos, M., \& Rosenthal, D. (2016). Neural Antecedents of Spontaneous Voluntary Movement: A New Perspective. Trends in Cognitive Sciences, 20(2), 77-79. https://doi.org/10.1016/j.tics.2015.11.003

Schurger, A., Sitt, J. D., \& Dehaene, S. (2012). An accumulator model for spontaneous neural activity prior to self-initiated movement. Proceedings of the National Academy of Sciences, 109(42), E2904-E2913. https://doi.org/10.1073/pnas.1210467109

Smith, P. L., \& Ratcliff, R. (2004). Psychology and neurobiology of simple decisions. Trends in Neurosciences, 27(3), 161-168. https://doi.org/10.1016/j.tins.2004.01.006

Stamm, J. S. (1985). The uncertainty principle in psychology. Behavioral and Brain Sciences, 8(4), 553-554. https://doi.org/10.1017/S0140525X0004509X

Thorpe, S., Fize, D., \& Marlot, C. (1996). Speed of processing in the human visual system. Nature, 381(6582), 520-522. https://doi.org/10.1038/381520a0

Uchida, N., \& Mainen, Z. F. (2003). Speed and accuracy of olfactory discrimination in the rat. Nature Neuroscience, 6(11), 1224-1229. https://doi.org/10.1038/nn1142

Usher, M., \& McClelland, J. L. (2001). The time course of perceptual choice: The leaky, competing accumulator model. Psychological Review, 108(3), 550-592. https://doi.org/10.1037/0033-295x.108.3.550

van Gaal, S., \& Lamme, V. A. F. (2012). Unconscious high-level information processing: Implication for neurobiological theories of consciousness. The Neuroscientist: A Review Journal Bringing Neurobiology, Neurology and Psychiatry, 18(3), 287-301. 